\title{
Hacer sociología de la sociología. Los aportes de Raymond Aron a la sistematización del pensamiento sociológico
}

\section{Doing sociology of sociology. The contributions of Raymond Aron to the systematization of sociological thought} Anabel Beliera

Laboratorio de Estudios en Sociología y Economia del Trabajo - Instituto de Investigaciones en Ciencias Sociales y Humanidades (Universidad Nacional de La Plata-CONICET), Argentina

\section{RESUMEN:}

Al sistematizar la teoría sociológica, Aron ([1967] 1996a, 1996b) evitó ordenar a los autores de manera armónica como si las ideas se establecieran con grados crecientes de consenso y desarrollo a lo largo de la historia, y eligió en cambio mostrar las diversas concepciones de pensadores que se propusieron definir lo social y estudiarlo de manera científica. Su intención no es establecer un nuevo canon de autores, sino pensar las tensiones constitutivas de esta ciencia de lo social. En este artículo propongo retomar la concepción de Aron sobre la relación entre historia y teoría para comprender su propia propuesta teórica y entenderla en relación al contexto académico del que participó. Asimismo, evaluaré la recepción de su teoría en la sociología enseñada en el siglo XXI en Argentina.

Palabras Clave: Aron, Teoría sociológica, Historia, Epistemología.

\section{Abstract:}

In systematizing sociological theory, Aron ([1967] 1996a, 1996b) escapes to order the authors harmoniously as if ideas were established with increasing degrees of consensus and development throughout history, and chose instead to show the different conceptions of authors who sought to define what social and study it in a scientific manner. His intention is not to establish a new canon of authors but to rethink the constitutive tensions of sociology. In this article I propose to take up Aron's conception of the relationship between history and theory in order to understand his own proposal for the systematization of sociological theory, and to understand it in relation to the academic context in which he participated. Likewise, I will evaluate the reception of his social theory in the sociology taught in the 21 st century in Argentina.

KEYWORDS: Aron, Sociological theory, History, Epistemology.

\section{INTRODUCCIÓN}

Aron afirma que a medida que exploran sus objetos de investigación, los sociólogos no pueden eludir interrogarse simultáneamente sobre qué es la sociología. Esto se vincula con el contexto académico en el que participó este autor, en el que sociólogos de muy diversas tradiciones (presentados por él en dos polos, los sociólogos norteamericanos y los sociólogos soviéticos) reivindicaban para la sociología tareas casi opuestas: los primeros reivindicaban una sociología analítica y empírica que "multiplica las encuestas mediante cuestionarios y entrevistas, con el fin de determinar cómo viven, piensan, sienten y juzgan los hombres sociales, o si se prefiere, los individuos socializados", mientras que los segundos exigían a la sociología que fuera "sintética -o global-, histórica y determinista"; adoptaban un "enfoque total" que "abarcaba al conjunto de la sociedad, aprehendida en su movimiento" (Aron, [1967] 1996a, p. 10). Denomina estas posiciones caricaturizadas como sociografía e ideología.

Aron (1969) reconoce que las diferencias que separan a estas tradiciones no eran nuevas, sino que estaban implícitas entre los fundadores de la sociología, quienes le atribuían dos características a esta nueva ciencia: por un lado, el rigor científico (la preocupación por el método y la insistencia en la demostración); por otro 
lado, su objeto de estudio (tratar de captar fenómenos sociales totales con carácter global). En la conjunción de los dos propósitos presentes en esta concepción (ser una ciencia particular pero que aborde los fenómenos sociales totales) resultan los conflictos entre diversas tradiciones: la sociología está siempre dividida entre estas dos intenciones (científica por una parte y sintética por la otra), y, según los países, los momentos y las escuelas, se abona la intención científica y multiplica las encuestas de detalle o, por el contrario, quiere mantener la intención sintética y corre el peligro de perderse en la filosofía (Aron, 1969).

¿Qué elementos articula la perspectiva cognitiva de esta ciencia que permiten que teóricos de tan diversas perspectivas reclamen para sí el status de sociólogos? Aron afirma que "la sociología es el estudio que se pretende cientifico de lo social como tal ${ }^{1}$, sea en el nivel elemental de las relaciones interpersonales, o en nivel macroscópico de los grandes conjuntos, las clases, las naciones, las civilizaciones" (Aron, [1967] 1996a, p. 16). Pero esta conclusión es sólo parcial, pues lleva necesariamente a realizar una autointerrogación respecto de qué implica adoptar una perspectiva científica sobre lo social como tal. Aron propone buscar la respuesta en la historia, pues le resulta imposible comprender la perspectiva sociológica sin reponer los aportes que realizaron diversos autores en diferentes momentos del pasado ${ }^{2}$. Para Aron ([1967] 1996a) resulta evidente que los sociólogos modernos sean herederos de quienes los precedieron, pero no busca reconstruir un conocimiento continuo sin contradicciones ni impone a esta disciplina una tendencia armonizada. Tal como ha sido señalado por Adorno (1996), si aceptamos el hecho de que la sociedad es inminentemente contradictoria, no resulta sorprendente que la ciencia que se ocupa de la sociedad no posea una continuidad lineal.

Aron (1969) considera que hay tres modos de desarrollo de la teoría sociológica: en primer lugar, la sociología se desarrolla por una acumulación de hechos (hay un progreso del conocimiento científico porque se conocen más hechos y se los conoce mejor); en segundo lugar, se desarrolla por la renovación de los problemas y por la posición de nuevas preguntas; sólo en tercer lugar, la sociología se desarrolla por el progreso de la teoría, en donde se afinan los instrumentos conceptuales gracias a los cuales tratamos de comprender la realidad social. Sin embargo, considera que no existe un sistema de conceptos universalmente válidos, o "por lo menos, es posible que el sistema de conceptos universalmente válidos esté formalizado a tal punto, que deje de ser un instrumento adaptado al análisis de las sociedades concretas" (Aron, [1967] 1969, p. 11). Por ende, se niega a presentarnos a los autores como si las ideas se ordenaran, con grados crecientes de consenso y desarrollo, constituyendo una unidad de las ideas sociológicas desde sus comienzos hasta la actualidad. Nos introduce la teoría sociológica como una problemática de carácter dinámico e históricamente constituida: se trata del producto de un esfuerzo que realizaron diversos autores por comprender sus sociedades.

Las etapas del pensamiento sociológico (Aron, [1967] 1996a,1996b) es una obra fundamental en este sentido: su importancia radica en la demostración de que el pensamiento sociológico no existe en singular y que, en consecuencia, es bien difícil discernir sus etapas (Busino, 1986). En esta obra el autor afirma que la sociología pretende determinar los tipos fundamentales de la organización social, la lógica subterránea de la vida en común. Nosotros podemos afirmar que Aron realiza pues una "sociología de la sociología": estudia las características comunes de las corrientes sociológicas que considera opuestas para determinar la lógica subterránea que las une.

En este artículo realizaremos una revisión de los elementos centrales de su teoría sociológica. En primer lugar, revisaremos su concepción de la relación entre la historia y la teoría. En segundo lugar, presentaremos algunos puntos importantes de la exposición conceptual que Aron realiza y de la selección de autores que nos presenta. Finalmente, nos proponemos revisar la presencia curricular de los aportes de este autor en los programas de las carreras de sociología actualmente existentes en la Argentina, evaluando su recepción en la sociología enseñada en el siglo XXI en nuestro país. 


\section{Historia Y TEORÍA}

La concepción de la historia de Aron, sistematizada en su tesis doctoral (Aron, 1937), es básica en la comprensión de su teoría social (Lassalle, 2004)․ La misma se puede resumir en la fórmula "el hombre está en la historia, el hombre es histórico; el hombre es una historia".

En primer lugar, desde una posición crítica frente al positivismo, afirma que no hay ninguna verdad absoluta sino verdades parciales: la realidad social no es ni incoherente ni total, lleva en sí multiplicidades de órdenes parciales. Para Aron, la historia no está escrita de antemano ni determinada por una fatalidad imprevisible, sino que es imprevisible dado el carácter singular de todo acontecimiento, que debe ser comprendido desde las intenciones y las acciones de sus protagonistas (Aguilar, 2005; Molina, 2005). Reivindica un papel activo para el individuo, y proclama su libertad y capacidad creadora (Guibernau Montserrat, 1994).

En segundo lugar, afirma que los hombres sólo pueden comprender su posición en el mundo después de reconocer el devenir real del que sólo son un átomo (Aron, 1985b), de modo que están "en", y mediante el momento histórico al que pertenecen (Lassalle, 2004). Esta condición histórica influye también en el quehacer científico, pues "el sujeto en busca de la verdad objetiva [también] está inmerso en la materia que quiere explorar y que lo penetra" (Aron, 1985b, p. 52). Por ende, busca comprender su época "tan honestamente como fuera posible, sin perder jamás la conciencia de las limitaciones del saber” (Aron, 1985b, p. 52).

En tercer lugar, para Aron la experiencia humana está marcada por el intento de comprender cómo vivieron nuestros antepasados, por qué y cómo en un determinado lugar acontecieron ciertos hechos -respecto de los cuales resulta básica la comprensión de las intenciones de los actores que las protagonizaron-, y cuál fue el sentido de esos hechos a partir de la reconstrucción de conjuntos inteligibles de la realidad (Lassalle, 2004, p. 195). De acuerdo Aron ([1967] 1996a, p. 16), el deber del "buen historiador" es conservar "el sentido de la especificidad de cada época, de la sucesión de las épocas y finalmente de las constantes que son las únicas que autorizan a hablar de una sola y misma historia”. El estudio de la historia siempre implicará tomar conciencia de la condición del hombre dentro de un mundo incoherente que vive desgarrado (Lassalle, 2004).

Afirma que el método "comprensivo" debe ser el punto de partida para el estudio de la historia, pues se debe captar lo singularmente irrepetible comprendiendo al hombre como un sujeto histórico -y no como un espíritu puro o un yo trascendente- (Lassalle, 2004) ${ }^{4}$. Trata de comprender la realidad a partir de los valores que mueven a la acción a los sujetos en el marco histórico-social en el que se encuentran. Busca captar el sentido de lo vivido comprendiendo la infinidad de elecciones e introspecciones que dan origen a los procesos sociales. Por ende, cualquier reconstrucción histórica guarda para Aron un carácter inacabado, ya que nunca se es capaz de deducir todas las relaciones. Nuestro saber sobre las sociedades está en permanente revisión: por un lado, porque no hay un saber último debido a la relatividad del punto de vista adoptado por el observador -que Aron teorizó en línea con la teoría weberiana-, y por otro lado, porque está abierto a la crítica -es decir, no es dogmático- (Maestre, 2005).

Veamos pues en qué nos ayuda la concepción de la historia de Raymond Aron para comprender su teoría social. En primer lugar, dado que Aron enfatiza que "los hombres hacen la historia, aunque no sepan qué historia están haciendo", debemos comprender su reconstrucción de la teoría sociológica como un intento de analizar diversos autores que "hicieron la sociología", aunque no supieran que la estaban haciendo. Aron ([1967] 1996a) organiza una galería de retratos intelectuales que alimentaron la sistematización de herramientas conceptuales y métodos para abordar de forma científica lo social como tal. Los autores que nos presenta reivindicaron métodos empíricos, realizaron encuestas mediante muestreos, aplicaron sus propios sistemas conceptuales, interrogaron de cierto ángulo la realidad social, exhibieron un enfoque especifico (Aron, [1967] 1996a). Por más que los autores se ordenen cronológicamente a lo largo de la historia, eso no significa que uno venga antes o después que el otro en un camino imaginario que empezaría por los orígenes 
de la sociología y terminaría en nuestros días. Aron se reúsa a dividir a los autores precursores de los padres fundadores o clásicosde la sociología, y prefiere presentárnoslos de acuerdo a las herramientas metodológicas y teóricas que elaboraron para comprender su sociedad y su tiempo.

En este sentido, aunque resulte extraño para la organización canónica de autores en nuestra disciplina, Aron nos presenta en el primer tomo de su libro a Montesquieu, Comte, Marx o Tocqueville, y en el segundo tomo a Durkheim, Pareto y Weber. Debe resaltarse pues que los autores incluidos en el primer tomo no revisten para Aron un carácter de precursores o fundadores, sino de sociólogos -al igual que los incluidos en el segundo tomo-. La reconstrucción que nos propone no se orienta a buscar los orígenes de la sociología moderna, sino a presentarnos sus tensiones constitutivas. La pertenencia de un autor del terreno de la sociología no se debe a que haya creado el término sociología o haya justificado su diferenciación respecto de las otras ciencias sociales, sino a que orientaron su acción a la intención específica de esta disciplina: conocer científicamente lo social como tal. Y si ha de definirse en estos términos, "Montesquieu es entonces, en mi opinión, un sociólogo con el mismo derecho que Augusto Comte” (Aron, [1967] 1996a, p. 29).

En segundo lugar, entender su concepción de la historia nos permite comprender sus intenciones respecto a la reconstrucción de la historia de la disciplina sociológica. Aron ([1967] 1996a, 1996b) no sólo repone las preocupaciones de los autores analizados, sino que estudia la relación entre sus conceptualizaciones teóricas y el contexto en el que vivieron, y finalmente busca dar sentido a esa historia sistematizando la construcción de diversas herramientas que forjaron el conocimiento científico de lo social. Aron aborda "las etapas del pensamiento sociológico" a partir del método "comprensivo": trata de comprender a los autores a partir de los valores y sentidos que orientaron sus acciones en el marco histórico-social en el que se encontraron. Busca captar el sentido de lo vivido, analizando tanto los acontecimientos a los que se vieron expuestos como sus elecciones y tomas de posición. En ese sentido, el criterio de selección de los autores que nos presenta es doble: se trata de autores que pensaron su tiempo y que simultáneamente produjeron herramientas potentes para analizarlas (Aron, [1967] 1996a).

En tercer lugar, como cualquier reconstrucción histórica, no se trata aquí de una verdad absoluta. No le falta sinceridad intelectual a Aron al afirmar que los autores incluidos en sus libros responden a sus intereses personales y a su propia formación. En este sentido, comienza presentando a Montesquieu no sólo por razones epistemológicas, sino porque "había consagrado antes un curso de un año completo" a este autor (Aron, [1967] 1996a, p. 19) , y a Tocqueville porque le despierta gran interés la participación de este autor liberal en el contexto de los grandes debates políticos de su época. Asimismo, la presentación de los autores está marcada por sus afinidades teóricas: el fuerte debate que mantuvo con los marxistas de su época, su afinidad por la teoría weberiana, su rechazo al colectivismo durkheimniano francés. Dado que considera que ninguna reconstrucción histórica es capaz de deducir todas las relaciones ni agotar todas las significaciones, nos aclara que la reconstrucción de los autores que nos presenta guarda un carácter inacabado.

\section{El pensamiento sociológico de Raymond Aron}

Para comprender la teoría sociológica aroniana -y cualquier otra- debemos comprender la posición del autor en el campo de relaciones en el que estaba inmerso, los debates académicos y políticos en los que participó, y los interrogantes que lo motivaron.

En este caso, debemos tener en cuenta que las casi ocho décadas de siglo que vivió -entre los años 1905 y 1983- lo colocaron en un escenario histórico amenazado por el totalitarismo y el horror bélico (Lassalle, 2004). Aron escribe sus trabajos iniciales mientras se produce la anexión nazi de Austria; deduce de los difíciles momentos que le toca vivir la idea de que la inteligibilidad de la historia es siempre fragmentaria y elusiva de cualquier aproximación totalizadora. Se percibe en los trabajos iniciales de este autor un "rechazo metodológico hacia cualquier tipo de visión globalizadora el mundo histórico, oponiendo a ella una 
propuesta analítica basada en la moderación, el diálogo y un argumentario que funda en la 'acción razonable' como criterios operativos" (Lassalle, 2004, p. 189).

Sin embargo, esto no lleva a identificar sociología y política. Si bien consideraba conveniente que los sociólogos no carecieran de interés político, pensaba que esto no definía a la sociología en sí misma -sino a todas las ciencias sociales-, y creía que los sociólogos deberían ser capaces de sublimar sus intereses políticos particulares. Considera que el verdadero peligro es que los sociólogos estudien "los aspectos agradables de las sociedades que prefieren, y los aspectos desagradables de las sociedades que no les son simpáticas". Es decir, considera que "el verdadero peligro es la parcialidad no reconocida" (Aron, 1969, p. 12).

Debe entenderse esta postura en el contexto de discusiones del que Aron participa, especialmente con el marxismo que en ese momento dominaba el mundo intelectual europeo (Guibernau Montserrat, 1994). La obra de Marx ejerció una notable atracción en la obra de Aron, aunque mantuvo con esta teoría una evaluación crítica $^{5}$, que se orientaba a poner de manifiesto la disociación entre la teoría marxista y la experiencia histórica (Lassalle, 2004). Estas críticas le sirvieron para firmar su condena académica en un momento en que el régimen soviético ejercía fascinación entre los sectores de la intelectualidad progresista (Guibernau Montserrat, 1994). Los debates con el marxismo de su época cristalizaron en la confrontación entre Aron y Sartre, quienes habían sido cercanos amigos durante la juventud, pero se fueron distanciando progresivamente merced a sus diferencias políticas y académicas. ${ }^{6}$

Pero el descubrimiento del mundo de la política en su biografía no se data en a la década del 60 sino en la década del 30, a partir de su estancia juvenil en Alemania (1930-1931 y 1931-1933). Fue allí cuando comenzó a dedicarse al estudio de los fenómenos políticos a partir de la observación racional y desencantada de este universo contradictorio por definición, habitado por el interés y las pulsiones (Campi, 2005). Producto del trabajo realizado estos años escribió La sociología alemana contemporánea (Aron, 1935), donde recorrió el pensamiento de los grandes pensadores alemanes -entre otros: Weber, Simmel, Tonnies, Oppenheimer, Scheler, Mannheim-. La repercusión formativa más importante tuvo lugar en contacto con la obra de Max Weber, de quien admiraba la capacidad de compatibilizar en su quehacer reflexivo tanto la experiencia histórica como la comprensión de la política (Lassalle, 2004).

Se posiciona del lado del paradigma de la sociología de la acción, cuestión que explica tanto sus gustos y preferencias por la sociología alemana como su débil intimidad de pensamiento durkheimniano (Busino, 1986). Él mismo confiesa: "[temo] haber sido injusto con respecto a Emile Durkheim, hacia cuyo pensamiento siempre experimenté una antipatía inmediata" ${ }^{7}$, debido al "sociologismo sobre el cual desembocan tan a menudo los análisis sociológicos y las intuiciones profundas” de este autor (Aron, [1967] 1996a, p. 22). Entiende por sociologismo a "la voluntad de explicar todos los fenómenos incluso espirituales, moral o conocimiento, a partir de la sociedad" (Aron, 1969, p. 6). Por el contrario, Aron reivindica un papel activo para el individuo en la formación de las organizaciones sociales, y proclama su libertad y capacidad creadora (Guibernau Montserrat, 1994).

Aron es fuertemente influido por la obra de Tocqueville, de quien rescata la explicación no doctrinaria de los fenómenos políticos. La democracia no aparece en la obra de este autor como un régimen que deba ser definido en el plano de las ideas, sino a través de las instituciones y del sistema o régimen político realmente existente (Maestre, 2005). Ambos conciben una historia abierta -aunque admiten una cierta dirección o sentido en el devenir de la humanidad, que prevén enfocado hacia la consolidación de las sociedades democráticas-.

Con sus conceptualizaciones Aron nos invita a pensar que no debe entenderse por sociología un dominio del saber delimitado a partir de reglas metrológicas fijas, competencias cognitivas definidas y bordes disciplinares delimitados, sino más bien como una manera de aprender la realidad social, un medio -válido como otros- para dar cuenta de la sociedad y su devenir (Busino, 1986). No deberíamos buscar en Las etapas del pensamiento sociológico un canon de autores estabilizados y fijos, sino una reconstrucción de diversos autores que buscaron aprender su realidad social y generar herramientas conceptualizarla. Podemos 
concederle a Aron el mismo honor: no debemos abordar su teoría como un cuerpo de conceptos y autores fijos y delimitados, sino como una reconstrucción realizada a partir de sus preocupaciones sociales y políticas, en el marco del contexto histórico y académico en que produjo.

\section{Aron en Argentina}

En este apartado me ocuparé de analizar la presencia curricular de los aportes de Aron en la sociología enseñada en el siglo XXI en Argentina. Para ello, he revisado y comparado los programas de las materias de teoría social de once carreras de sociología actualmente existentes en las universidades nacionales ${ }^{8}$.

Siguen algunas aclaraciones metodológicas. En primer lugar, se analizan únicamente los programas correspondientes a las materias curriculares de los planes de estudio, no así los cursos introductorios, nivelatorios, o ciclos básicos comunes. En segundo lugar, he analizado únicamente las materias obligatorias de cada una de las carreras, y no aquellas que revisten carácter de optativas o seminarios específicos. En tercer lugar, dada la ineludible articulación entre los contenidos de Teoría Sociológicarespecto a los de Historia del Pensamiento Sociológico, hemos decidido incluir en el análisis los programas de las materias destinadas a la enseñanza de ambas. En cuarto lugar, debemos aclarar que hemos realizado la búsqueda de los programas en los sitios de internet de las propias facultades, institutos o departamentos de Sociología ${ }^{9}$, y, en los casos que eso no fue posible, realizamos un rastreo de los programas a través del contacto con docentes de cada una de las universidades ${ }^{10}$. Asumimos que el último programa publicado en internet se corresponde con los contenidos actualmente dictados en las materias, y en cada caso detallamos el año del programa analizado. Finalmente, deben hacerse aclaraciones respecto de dos universidades nacionales: respecto de la carrera de Sociología de la UNC, es una carrera de reciente creación, por lo que sólo se han instaurado hasta el momento las materias del primero y segundo año correspondientes al plan de estudios. Respecto de la UNVM, a finales del año 2017 se aprobó un cambio en el plan de estudios, que comenzó a implementarse en el año 2018, por lo que la mayoría de las materias no contaba con los programas de contenidos. Para las materias que comenzaron a dictarse en el año 2018, analizamos el programa actual. Para las materias que comenzarían a dictarse en los años posteriores, pero que tenían una correspondencia con alguna de las materias preexistentes, analizamos el programa correspondiente a la materia anterior. Sólo el caso de la materia Teoría Social III -que comenzaría en el año 2021-, no pudimos incorporarlo en el análisis.

Se han analizado en total 49 programas de las diferentes materias de Teoría Sociológica. En el cuadro presentado a continuación podrá observarse el listado de programas analizados, el año los mismos, y la ausencia o presencia (en la bibliografía obligatoria u optativa) de la teoría sociológica de Raymond Aron respecto de cada uno de ellos. 


\begin{tabular}{|c|c|c|c|c|c|c|c|c|}
\hline \multirow{2}{*}{\begin{tabular}{|l|} 
Carreras de \\
Sociologia en \\
Universidades \\
Nacionales \\
Argentinas
\end{tabular}} & \multirow{2}{*}{\multicolumn{2}{|c|}{ Programas de materias }} & \multicolumn{2}{|c|}{ Programas } & \multirow[b]{2}{*}{ Año } & \multicolumn{3}{|c|}{$\begin{array}{l}\text { Teoria Social de R. } \\
\text { Aron }\end{array}$} \\
\hline & & & si & no & & Ausencia & Obl. & Ampl. \\
\hline \multirow{7}{*}{$\begin{array}{l}\text { Universidad } \\
\text { de Buenos } \\
\text { Aires }\end{array}$} & 1 & Sociología General-cátedra Rubinich- & $x$ & & 2018 & $x$ & & \\
\hline & 2 & Sociología General -cátedra Forte- & $x$ & & 2018 & $x$ & & \\
\hline & 3 & $\begin{array}{l}\text { Historia del Conocimiento Sociológico } \\
\text { I - cátedra Jenkins- }\end{array}$ & $x$ & & 2018 & & & $\mathrm{x}$ \\
\hline & 4 & $\begin{array}{l}\text { Historia del Conocimiento Sociológico } \\
\text { I - cátedra Fernández- }\end{array}$ & $x$ & & 2018 & $x$ & & \\
\hline & 5 & $\begin{array}{l}\text { Historia del Conocimiento Sociológico } \\
\text { II -cátedra Aronson- }\end{array}$ & $\mathrm{x}$ & & 2017 & $\mathrm{x}$ & & \\
\hline & 6 & $\begin{array}{l}\text { Historia del Conocimiento Sociológico } \\
\text { II -cátedra Raus- }\end{array}$ & $x$ & & 2016 & & & $\mathrm{x}$ \\
\hline & 7 & Sociología Sistemática & $x$ & & 2017 & & $x$ & \\
\hline \multirow{4}{*}{$\begin{array}{l}\text { Universidad } \\
\text { Nacional de } \\
\text { Córdoba }\left(1^{\circ} \mathrm{y}\right. \\
2^{\circ} \text { año) }\end{array}$} & 1 & $\begin{array}{l}\text { Introducción al Conocimiento de las } \\
\text { Ciencias Sociales }\end{array}$ & $x$ & & 2017 , & $x$ & & \\
\hline & 2 & Sociología Sistemática & $x$ & & 2018 & $x$ & & \\
\hline & 3 & Teoría Sociológica 1 (Clásica) & $x$ & & 2017 & & $x$ & \\
\hline & 4 & \begin{tabular}{|l|} 
Teoría Sociológica 2 \\
(Contemporánea)
\end{tabular} & $x$ & & 2018 & $x$ & & \\
\hline \multirow{4}{*}{$\begin{array}{l}\text { Universidad } \\
\text { Nacional de } \\
\text { Cuyo }\end{array}$} & 1 & $\begin{array}{l}\text { Formación del Pensamiento } \\
\text { Sociológico }\end{array}$ & $x$ & & 2018 & & & $\mathrm{x}$ \\
\hline & 2 & Teoría Social Clásica & $x$ & & 2018 & & & $x$ \\
\hline & 3 & Teoría Sociológica Contemporánea & $x$ & & 2017 & $x$ & & \\
\hline & 4 & Sociología Sistemática & $\mathrm{x}$ & & 2017 & $\mathrm{x}$ & & \\
\hline \multirow{4}{*}{$\begin{array}{l}\text { Universidad } \\
\text { Nacional de } \\
\text { La Plata }\end{array}$} & 1 & Sociología General & $x$ & & 2018 ; & $\mathrm{x}$ & & \\
\hline & 2 & Teoría Social Clásica 1 & $x$ & & 2018 & & & $x$ \\
\hline & 3 & Teoría Social Clásica 2 & $x$ & & 2014 & & & $x$ \\
\hline & 4 & Teoría Social Contemporánea & $x$ & & 2018 & $x$ & & \\
\hline \multirow{5}{*}{$\begin{array}{l}\text { Universidad } \\
\text { Nacional de } \\
\text { Mar del Plata }\end{array}$} & 1 & Introducción a la Sociología & $x$ & & 2018 & $x$ & & \\
\hline & 2 & Sociología Sistemática & $x$ & & 2018 & $x$ & & \\
\hline & 3 & Teoría Sociológical & $x$ & & 2012 & $x$ & & \\
\hline & 4 & Teoría Sociológicall & $x$ & & 2012 & $x$ & & \\
\hline & 5 & Teoría Sociológicalll & & $x$ & & & & \\
\hline \multirow{5}{*}{$\begin{array}{l}\text { Universidad } \\
\text { Nacional de } \\
\text { San Juan }\end{array}$} & 1 & $\begin{array}{l}\text { Curso de iniciación: Introducción a las } \\
\text { Ciencias Sociales }\end{array}$ & & $x$ & & & & \\
\hline & 2 & Teoría Sociológical & $x$ & & 2011 & & $\mathrm{x}$ & \\
\hline & 3 & Teoría Sociológicall & $x$ & & 2011 & $\mathrm{x}$ & & \\
\hline & 4 & Teoría Sociológica III & $x$ & & 2009 & $\mathrm{x}$ & & \\
\hline & 5 & Teoría Sociológica IV & $x$ & & 2009 & $x$ & & \\
\hline \multirow{3}{*}{$\begin{array}{l}\text { Universidad } \\
\text { Nacional de } \\
\text { Santiago del } \\
\text { Estero }\end{array}$} & 1 & Introducción a la Sociología & $x$ & & 2010 & $x$ & & \\
\hline & 2 & Historia del Pensamiento Sociológico & $x$ & & 2012 & & & $\mathrm{x}$ \\
\hline & 3 & Teoría Sociológical & $x$ & & $2010 \mid$ & & & $x$ \\
\hline \multirow{6}{*}{$\begin{array}{l}\text { Universidad } \\
\text { Nacional de } \\
\text { Tierra del } \\
\text { Fuego }\end{array}$} & 1 & $\begin{array}{l}\text { Introducción al Estudio de la } \\
\text { Sociedad, la Cultura y el Ambiente }\end{array}$ & $\mathrm{x}$ & & 2018 & $x$ & & \\
\hline & 2 & Teoría Sociall & $x$ & & 2018 & $x$ & & \\
\hline & 3 & Teoría Socialll & & $x$ & & & & \\
\hline & 4 & Teoría Social III & $x$ & & 2018 & $x$ & & \\
\hline & 5 & Conflicto y Cambio Social & $x$ & & 2018 & $x$ & & \\
\hline & 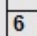 & Fundamentos de Sociología & & $x$ & & & & \\
\hline
\end{tabular}




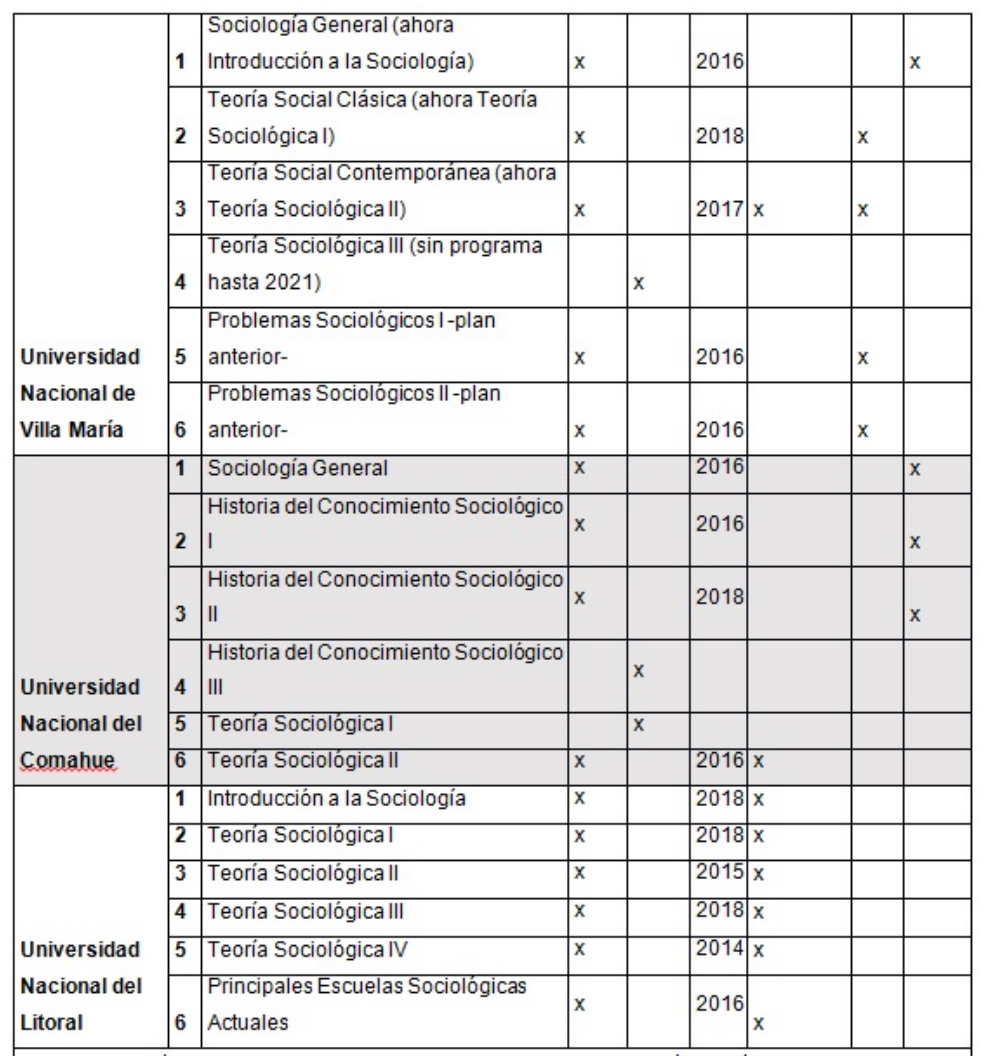

Elaboración propia con base en programas de materias obligatorias de Teoría Sociológica impartidas en el siglo XXI en las universidades nacionales argentinas

Es notoria la escasa presencia de este autor en las carreras de sociología actualmente existentes en nuestro país. Su teoría está ausente en todos los programas de las materias impartidas en las UNMDP, UNTF y UNL. Respecto de las universidades en las que se abordan algunos de los aportes de este autor, debe decirse que se trata de pocas materias: sólo el 30,6 \% de los programas analizados (15 de los 49). De estas 15 materias, sólo en tres programas se trata de un contenido correspondiente a la bibliografía obligatoria: son los casos de la cátedra de Sociología Sistemática de la UBA, Teoría Social Clásica de la UNC, y Teoría Sociológica I de la UNSJ. En las doce materias restantes (correspondientes a las carreras de la UBA, UNCU, UNLP, UNSE, UNCo, UNVM) forma parte de la bibliografía ampliatoria.

Salvo en una materia en la que se referencia el libro La sociología alemana de Aron (programa de Sociología sistemática de la UBA), en los programas analizados sólo se indica la lectura de la obra Las etapas del pensamiento sociológico (14 programas). En 8 de estas materias, esta obra aparece referenciada de manera general, sin especificarse ningún capítulo especial ni una sección de páginas determinada ${ }^{11}$. Incluso sólo en la mitad de estos casos se aclara en la referencia bibliográfica que se trata de una obra con dos tomos. Suponemos pues que esta obra no es abordada para hacer un análisis de los aportes de Aron en sí mismos, sino como un marco general para contextualizar las obras de los autores abordados en estas materias (Montesquieu, Comte, Marx, Tocqueville, Durkehim, Pareto, Weber), y que se supone que el estudiante seleccionará los fragmentos que se corresponden con los contenidos enseñados.

En los seis casos en que se especifica la lectura de un capítulo o sección determinada se delimita la lectura de la sistematización que Aron realiza de las teorías de Comte (bibliografía ampliatoria de Teoría Social Clásica de la UNLP), Emile Durkheim (bibliografía obligatoria de Teoría Sociológica 1 de la UNC y bibliografía ampliatoria de las materias Teoría Social Clásica de la UNLP, Sociología General de la UNVM e Histórica del Conocimiento Sociológico I de la UNCo) y Max Weber (bibliografía ampliatoria de las materias Teoría Social Clásica de UNCU e Historia del Conocimiento Sociológico II -cátedra Raus- de la UBA). 
Debido a que algunos los autores abordados por Aron ([1967]1996a, [1967] 1996b) suelen formar parte de los autores canónicos de la ciencia política y no necesariamente de la teoría sociológica-como Tocqueville y Montesquieu-, hemos revisado también los programas de introducción a los conceptos de teoría política ${ }^{12}$. En todos los programas revisados la teoría social de Raymond Aron se encontraba ausente ${ }^{13}$.

De la información presentada se desprende que la presencia curricular de los aportes de Aron es escasa, si no nula, en la sociología enseñada en el siglo XXI en nuestro país. Seguramente esto responda tanto a cuestiones internas de la sociología argentina como a la propia trayectoria académica del autor, debido a que sus posiciones políticas y académicas lo confinaron a un trabajo solitario y aislado incluso del mundo académico francés, donde producía. Este autor ha tenido poca influencia en la sociología internacional en general -salvo en Alemania, donde se formó- (Baverez, 2000) , y especialmente en la sociología latinoamericana. Argentina no ha sido la excepción.

\section{Conclusiones}

A lo largo de este artículo hemos realizado una revisión de los elementos centrales de la teoría sociológica de Aron, a partir de su conceptualización de la relación entre la historia y la teoría, y de sus posicionamientos políticos y académicos en el contexto histórico en que vivió. Se propone realizar una revisión del pensamiento sociológico en la que nos muestra las tensiones filosóficas y epistémicas de autores que se propusieron definir lo social, analizar el consenso social y ubicar las diversidades históricas de prácticas que, por otro lado, se buscaba tipificar. Las etapas del pensamiento sociológico (Aron, [1967] 1996a, [1967] 1996b) es una obra fundamental en este sentido. Allí Aron realiza pues una "sociología de la sociología”: estudia las características comunes de las corrientes sociológicas que considera opuestas para determinar la lógica subterránea que las une.

La sociología es, para este autor, tanto una práctica sistemática como un cuerpo acumulado de conocimiento, pero no le exige una trayectoria científica continua sin contradicciones. Se reúsa a situar un punto cronológico que divida a los autores precursores de los padres fundadores o clásicos de la sociología, y prefiere presentarnos una galería de retratos intelectuales para aprehender lo esencial del pensamiento de diversos sociólogos que alimentaron la sistematización de herramientas conceptuales y métodos para abordar de forma científica lo social como tal. La selección de estos autores está orientada tanto por criterios epistemológicos como por la formación y las preferencias políticas y académicas de Raymond Aron: el fuerte debate que mantuvo con los marxistas de su época, su afinidad con la sociología alemana -y específicamente la teoría weberiana-, su rechazo al colectivismo durkheimniano francés, su vínculo con la teoría liberal.

Finalmente, en este artículo revisamos la presencia curricular de los aportes de Aron en los programas de las carreras de sociología actualmente existentes en la Argentina. Del análisis de los 49 programas analizados de once universidades nacionales, se desprende que la enseñanza de la teoría aronniana es escasa, si no nula. Sin embargo, cabe la pregunta, ¿por qué sería bueno enseñar la obra de Aron en los cursos básicos de teoría social de las carreras de sociología? Los aportes de este autor muestran de manera clara un aspecto que los estudiantes de sociología más tarde o más temprano deben comprender: la presencia de los autores no responde a ninguna cualidad esencial de los mismos ni a una reconstrucción histórica lineal y armónica, sino que está condicionada por múltiples debates epistemológicos y pedagógicos. A la vez, estos debates están marcados por la realidad histórica en que nos vemos inmersos y en la que participamos en la actualidad, tanto por las particularidades de nuestros países y de las realidades políticas que atravesamos, como del mundo académico - respecto de historia de nuestras carreras, de nuestra posición en el campo, la recepción y circulación de determinados autores-. Aron invita pues a que los estudiantes contextualicen a los autores en su tiempo y de acuerdo a las preocupaciones que los motivaron, y a que comprendan que nuestra propia mirada sobre ellos está marcada por nuestro tiempo y nuestras preocupaciones. 


\section{Bibliografía}

Adorno, T. W. (1996). Introducción a la sociología. (C. Gödde, Ed.). Barcelona: Gedisa.

Aguilar, E. (2005). Raymond Aron, veinte años después. Revista hispano cubana, (23), 97-104.

Aron, R. (1935). La sociologia alemana contemporánea. Paidos.

Aron, R. (1937). Introducción a la filosofía de la historia: ensayo sobre los limites de la objetividad histórica: completado con textos recientes. Paris : Gallimard.

Aron, R. (1969). Consideraciones generales sobre la sociología. Investigación Económica, 29(113), 75-86.

Aron, R. (1985a). Paz y guerra entre las naciones. 1, Teoría y sociología. Madrid: Alianza.

Aron, R. (1985b). Memorias. Madrid: Alianza Editorial.

Aron, R. (1996a). Las etapas del pensamiento sociológico. Tomo 1: Montesquieu, Comte, Marx, Tocqueville. Buenos Aires: Fausto.

Aron, R. (1996b). Las etapas del pensamiento sociológico. Tomo 2: Durkheim, Pareto, Weber. Buenos Aires: Fausto.

Bauman, Z., y May, T. (1994). Pensando Sociológicamente. Buenos Aires: Nueva Visión.

Baverez, N. (2000). Raymond Aron 1905-1983. Cités, (3), 251-258.

Busino, G. (1986). Raymond aron et la sociologie. L'Année sociologique (1940/1948), 36, 291-315.

Campi, A. (2005). Raymond Aron y la tradición del realismo político. En J. M. Lassalle (Ed.), Raymond Aron: un liberal resistente (pp. 69-94). Madrid: Fundación para el Análisis y los Estudios Sociales.

Gago, P. (2005). Raymond Aron frente a la intelligentsia. En J. M. Lassalle (Ed.), Raymond Aron: un liberal resistente (pp. 127-154). Madrid: Fundación para el Análisis y los Estudios Sociales.

García Vargas, J. (2005). Un atlantista a contra viento y marea. En J. M. Lassalle (Ed.), Raymond Aron: un liberal resistente (pp. 155-180). Madrid: Fundación para el Análisis y los Estudios Sociales.

Guibernau Montserrat, B. (1994). El pensamiento sociológico de Aron. El Ciervo: revista mensual de pensamiento y cultura, (516), 25-28.

Lassalle, J. M. (2004). Geometrías de un Sísifo liberal. El caso Aron. Cuadernos de Pensamiento Político, (2), 103-140.

Lassalle, J. M. (Ed.). (2005). Raymond Aron: un liberal resistente. Madrid: Fundación para el Análisis y los Estudios Sociales.

Maestre, A. (2005). Raymond Aron, un pensador político. En J. M. Lassalle (Ed.), Raymond Aron: un liberal resistente (pp. 139-160). Madrid: Fundación para el Análisis y los Estudios Sociales.

Molina, J. (2005). Sin ilusión, sin pesimismo. El realismo político liberal de Raymond Aron. En J. M. Lassalle (Ed.), Raymond Aron: un liberal resistente (pp. 261-304). Madrid: Fundación para el Análisis y los Estudios Sociales.

\section{Notas}

1 Cursivas en el original.

2 Esta tensión entre historia y teoría también ha marcado los debates pedagógicos. Como veremos, en las carreras de sociología en Argentina, es notable la oscilación entre las materias de historia del pensamiento sociológico y las denominadas teoría sociológica o sociologías sistemáticas. Estas dos formas de abordajes implican diversos criterios: por un lado, el de ordenar a los autores cronológicamente (precursores, clásicos, contemporáneos); por el otro lado, el de abordar la enseñanza de la sociología desde la presentación de problemas y conceptos (es decir, desde una perspectiva sistemática). Muchas de las carreras de sociología de nuestro país articulan ambos criterios.

3 Aron hizo su doctorado en Alemania, y su tesis fue defendida en 1937. El haber vivido en el contexto nazi en este país hizo que Aron asumiera una militancia antiautoritaria. Bajo estas coordenadas biográficas e históricas debe contextualizarse su preocupación intelectual liberal "de ver en la historia el resultado de las contribuciones individuales que los hombres aportan a su desarrollo mediante sus particulares acciones. De ahí su apuesta por la libertad y las instituciones liberales frente a los totalitarismos que negaban que la historia estuviera abierta al modelado que cada hombre impregna en ella con sus singulares decisiones" (Lassalle, 2005, p. 12). 
4 Como veremos más adelante, la adopción del método "comprensivo" se vincula con la influencia de la teoría weberiana luego de sus primeros años de formación en Alemania. Ver Aron (1985b).

5 Cuestión que no detallaremos in extenso, pues se aborda en este dossier por Sergio Morresi y Martín Vicente.

6 Para ampliar estos elementos, ver Aron (1985b).

7 Lo que no impide que lo reconozca como uno de los sociólogos más auténticos (Aron, 1969)

8 Se trata de las siguientes universidades: Universidad de Buenos Aires (UBA), Universidad Nacional de Córdoba (UNC), Universidad Nacional de Cuyo (UNCU), Universidad Nacional de la Plata (UNLP), Universidad Nacional de Mar del Plata (UNMDP), Universidad Nacional de San Juan (UNSJ), Universidad Nacional de Santiago del Estero (UNSE), Universidad Nacional de Tierra del Fuego (UNTDF), Universidad Nacional de Villa María (UNVM), Universidad Nacional del Comahue (UNCo) y Universidad Nacional del Litoral (UNL). Sólo ha restado analizar los programas de la carrera de Sociología de la Universidad Nacional de San Martín, debido a la imposibilidad de acceder a los mismos.

9 Son los casos de la UBA, UNC, UNCU, UNLP, UNL, UNC, UNCo, UNVM.

10 Por facilitarme los contactos y/o los programas de las materias requeridas, quiero agradecer la asistencia de German Soprano, Mariana Busso y Fernanda Torres de la UNLP; Julia Burton, Yamai Zapata, Mariana Giaretto, Dolores Sancho y Fernando Lizarraga de la UNCo; Fernando Aiziczon de la UNC, Jorge Jourdan, Gabriel Obradovich y Virginia Trevignani de la UNL; Matías Giletta, Mariano Schejter, Sofía Ruderman y Susana Roitman de la UNVM; Federico Lorenc Valcarce y María Laura Canestraro de la UNMDP.

11 Se trata de las materias Historia del conocimiento sociológico I de la UBA, Formación del pensamiento Sociológico de la UNCU, Teoría Social Clásica 2 de la UNLP, Teoría sociológica I de la UNSJ, Historia del Pensamiento Sociológico y Teoría Sociológica I de la UNSE, Sociología general e Historia del Conocimiento Sociológico II de la UNCo.

12 Se trata de los programas de Sociología Política de la UBA; Fundamentos de la ciencia política, Teoría Política 1 y 2 de la UNC; Sociología Política de la UNCU; Teoría Política y Sociología Política de la UNLP; Introducción a la Problemática Política de la UNMDP; Sociología política de la UNSJ; Sociología Política de la UNSE; Introducción al Pensamiento Político y Social, y Sociología Política de la UNTDF; Introducción a la Problemática Política de la UNVM; Teoría Política I y II, y Sociología Política I y II de la UNCo; Sociología Política de la UNL.

13 La única excepción era la materia Teoría Política de la UNLP, sin embargo, se trataba de un abordaje particular: en el desarrollo de las clases de trabajos prácticos se centraba en el estudio en profundidad de un tema específico, y, debido a que el tema abordado recientemente es "Paz y guerra en las naciones", está incluido en la bibliografía obligatoria el texto "Dialécticas de la paz y de la guerra" (Aron, 1985a). 